\title{
Asociación entre polimorfismos de la región promotora del gen del factor de necrosis tumoral alfa (TNF-alfa) con obesidad y diabetes en mujeres chilenas
}

\author{
José Luis Santos M 1,a, Ana Patiño G 2,b, Bárbara Angel B1,c, \\ José Alfredo Martínez $\mathrm{H}^{2, \mathrm{~d}}$, Francisco Pérez $\mathrm{B}^{1, \mathrm{~b}}$, Ana Claudia \\ Villarroel $\mathbf{B}^{1}$, Luis Sierrasesúmaga $A^{2}$, Cecilia Albala $B^{1}$. \\ Association between tumor necrosis \\ factor-alpha promoter polymorphisms \\ and type 2 diabetes and obesity \\ in Chilean elderly women
}

Background: Tumor necrosis factor-alpha (TNF-alpha) has an increased expression in the adipose tissue of obese subjects and is involved in insulin resistance. Aim: To screen for associations between -308G/A, -238G/A, -376G/A and -163G/A genetic variants of the TNF-alpha gene, diabetes and obesity-related variables. Material and methods: A group of 263 elderly women aged 60-90 years were recruited. Among them, an oral glucose tolerance test was performed and serum lipids measured in 100 women. TNF-alpha genotypes were determined by polymerase chain reaction (PCR) and analysis of restriction fragment lenght polymorphisms. Results: No significant differences were found when comparing allele frequencies in TNF-alpha polymorphisms of normal subjects with those having impaired glucose tolerance or type 2 diabetes. After excluding patients with previous diagnosis of diabetes, no significant differences by polymorphism carrier status were found for plasma levels of lipids, glucose and insulin. Additionally, no significant differences were found for the association between variables related to adiposity and the -308G/A polymorphisms. Conclusions: It is unlikely that polymorphisms in the promoter region of the TNF-alpha gene have a major influence in obesity and diabetes phenotypes in Chilean elderly women (Rev Méd Chile 2006; 134: 1099-106).

(Key words: Diabetes mellitus, type 2; Insulin resistance; Obesity; Tumor Necrosis Factor-alpha)

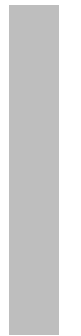

\footnotetext{
Recibido el 19 de octubre, 2005. Aceptado el 10 de marzo, 2006.

Trabajo financiado por FONDECYT \#1020703 y Línea Especial LE/97 de la Universidad de Navarra "Nutrición, Obesidad y Salud".

${ }^{1}$ Instituto de Nutrición y Tecnología de los Alimentos, Universidad de Chile. Santiago, Chile. ${ }^{2}$ Departmento de Fisiología y Nutrición y Laboratorio de Biología Molecular, Universidad de Navarra, Pamplona, España.

aPh.D. Biología. MSc Epidemiología Genética

${ }^{b} \mathrm{Ph} . \mathrm{D}$. Biología Molecular

cMatrona. MSc Biología de la Reproducción

dPh.D Farmacia
}

Correspondencia a: José Luis Santos. Instituto de Nutrición y Tecnología de los Alimentos, Universidad de Chile. El Líbano 5524. Casilla 138-11, Santiago, Chile. Teléfono: (56-2) 9781456. Fax: (56-2) 2214030. E mail: jsantos@inta.cl 
$\mathrm{E}^{1}$ factor de necrosis tumoral alfa (TNF-alfa) es una citoquina inducida en procesos inflamatorios producida principalmente por macrófagos ${ }^{1}$. La participación de TNF-alfa en la obesidad y la diabetes se ha puesto de manifiesto en modelos animales en los que se bloqueó la funcionalidad de esta citoquina ${ }^{2}$, así como en estudios donde se ha descrito una expresión incrementada de TNFalfa en el tejido adiposo de animales de experimentación con obesidad y en obesos humanos ${ }^{3}$. La relación entre una producción más alta de la TNF-alfa con el desarrollo de la resistencia a la insulina y diabetes, se ha apoyado en experimentos que han demostrado que TNF-alfa alteraría la señalización insulínica a nivel de sustrato insulínico 1, favoreciendo la aparición de resistencia a la acción de la insulina y el posterior desarrollo de diabetes tipo $2^{4,5}$. Varios polimorfismos simples (SNP's) se han descrito en su región promotora ${ }^{6}$, sugiriéndose que podría existir un mayor nivel de transcripción de TNF-alfa en portadores del alelo -308A comparado con la actividad transcripcional del alelo - $308 G^{7,8}$. Por ello, se esperańa que las variaciones genéticas en la región promotora de TNF-alfa pudieran jugar un papel en la obesidad y la diabetes.

El objetivo de este estudio es evaluar la asociación entre polimorfismos de la región promotora del gen TNF- alfa (-308G/A, -238G/A, -376G/A y -163G/A) con la obesidad y la diabetes en mujeres chilenas de edad avanzada.

\section{SUJETOS Y MÉTODO}

Sujetos. El presente estudio se planteó como un diseño de corte transversal, realizado en mujeres seleccionadas a partir de una muestra de base poblacional tomada en adultos mayores de 60 años, residentes en el gran Santiago ${ }^{9}$. A partir de esta muestra, se procedió a invitar nuevamente a mujeres en dos estudios que se realizaron entre los años 2001 y 2005.

Estudio 1: Se centró en la relación entre polimorfismos de TNF-alfa (-308G/A, -238G/A, -376G/A y -163G/A) con la diabetes tipo 2. Para evaluar este objetivo, se seleccionaron 100 mujeres (edad promedio $=67,6$ años; desviación estándar = 2,8 años; rango: 60-69 años).

Estudio 2: Se centró en la relación entre el polimorfismo -308G/A de TNF-alfa y la adiposi- dad. Para esta investigación se seleccionaron 263 mujeres (las 100 mujeres del estudio 1 más 163 mujeres adicionales), en las que se midieron diferentes variables antropométricas. La edad promedio de este grupo fue de 69,5 años (desviación estándar = 5,8 años; rango: 60-90 años).

Los participantes del estudio firmaron un consentimiento escrito que fue aprobado por el comité de ética del Instituto de Nutrición y Tecnología de los Alimentos (INTA) de la Universidad de Chile.

Antropometría y cálculo de grasa corporal. En todos los sujetos del estudio, se determinó peso y estatura para calcular el índice de masa corporal (IMC), así como el índice cintura-cadera, como medida de distribución de grasa corporal. Las variables de alto de rodilla, peso, perímetro de brazo, ancho de muñeca, dinamometría de mano y grosor de pliegue tricipital fueron medidas para estimar el agua corporal total y grasa corporal total, mediante el uso de ecuaciones antropométricas previamente desarrolladas y validadas en adultos mayores chilenos ${ }^{10,11}$. El índice de masa grasa (IMG) se calculó como el cociente entre la masa grasa en kilogramos dividido por la estatura en metros al cuadrado ${ }^{12}$.

Prueba oral de tolerancia a la glucosa y determinaciones clínicas de laboratorio. Se realizaron pruebas orales de tolerancia a la glucosa y determinaciones de laboratorio relacionadas con la resistencia a la insulina en las mujeres del estudio 1. Las muestras de sangre fueron tomadas entre las 7:00 y 9:00 h, después de una noche en ayuno. Tras la prueba oral de la tolerancia a la glucosa $(75 \mathrm{~g})$, las participantes se clasificaron como "diabetes tipo 2", "intolerancia a la glucosa" o "tolerancia a la glucosa normal" de acuerdo a los criterios de la Organización Mundial de la Salud ${ }^{13}$.

De las participantes del estudio 1, un grupo de 23 mujeres no realizaron la prueba de tolerancia a la glucosa completa por tener un diagnóstico previo de diabetes, y sólo se les tomó una muestra de sangre en ayunas. Otras 7 mujeres, que inicialmente desconocían su condición diabética, fueron clasificadas como diabéticas después de la prueba oral de la tolerancia a la glucosa. La elevada frecuencia de diabetes tipo 2 en esta 
muestra se debe, en nuestra opinión, a la existencia de un fenómeno de autoselección de personas que aceptaron participar en el estudio por tener un diagnóstico previo de diabetes. Aunque la existencia de autoselección actuaría como sesgo en la estimación de la prevalencia de diabetes tipo 2, este fenómeno no actuaría como sesgo en la comparación de frecuencias alélicas entre personas diabéticas y no-diabéticas. En el estudio 2 ( $\mathrm{n}=$ 263), un total de 39 mujeres conocía su condición diabética por un diagnóstico previo realizado por un médico.

Los niveles séricos de glucosa fueron determinados mediante la técnica de glucosa-oxidasa, mientras que los niveles de la insulina plasmática fueron medidos con radioinmunoensayo (DPC Diagnostic Corporation, USA). El colesterol plasmático, el HDLcolesterol y los niveles totales de los triglicéridos fueron determinados con métodos enzimáticos colorimétricos estandarizados (Boehringer Mannheim, Germany). Las concentraciones de insulina y glucosa plasmática en ayunas se utilizaron para calcular el índice de "Homeostasis Model Assessment" (HOMA) como indicador de resistencia a la insulina.

Determinación de genotipos. Se utilizó la reacción en cadena de la polimerasa (PCR) para amplificar segmentos específicos de la región promotora del gen TNF-alfa. Los ensayos de PCR fueron realizados en un termociclador Perkin-Elmer GeneAmp 2400, usando 150-250 ng de ADN genómico en un volumen final de reacción de $35 \mathrm{ml}$. La reacción de PCR contenía buffer 10x (Bioline, London, UK) y 1 U de Taq polimerasa, así como una concentración final de 0,5 mM de cada partidor, $200 \mathrm{mM}$ de cada dNTP y $2 \mathrm{mM}$ de $\mathrm{MgCl}_{2}$.

Para la determinación de los genotipos de los polimorfismos -308G/A y -238G/A, se amplificó un fragmento de 117 pares de bases usando los siguientes partidores: 5'- AGGCAATAGGTTTTGAGGGCCAT-3' (directo) y 5'-ACACTCCCCATCCTCCCGGCT-3' (reverso). Los nucleótidos subrayados difieren de la secuencia original, con el fin de crear, dos sitios de restricción reconocibles posteriormente por las enzimas NcoI y NLAIV. La PCR se inició con un paso de desnaturalización $\left(94^{\circ} \mathrm{C} / 5 \mathrm{~min}\right)$ seguido de 30 ciclos (desnaturalización a $94^{\circ} \mathrm{C} / 30 \mathrm{~s}$, hibridación a $61^{\circ} \mathrm{C} / 30 \mathrm{~s}$ y extensión a $72^{\circ} \mathrm{C} / 30 \mathrm{~s}$ ), con una extensión final de $72^{\circ}$ por $5 \mathrm{~min}$. Un volumen de $10 \mu \mathrm{l}$ del producto de PCR se incubó a
37으 durante $3 \mathrm{~h}$ con enzimas de restricción y se resolvió mediante electroforesis en geles de agarosa 3\% para la visualización de fragmentos. Específicamente, el polimorfismo -308G/A se determinó mediante la incubación con $2 \mathrm{U}$ de la enzima NcoI (New England Biolabs). En este polimorfismo, el genotipo homocigoto para el alelo salvaje $\mathrm{G}$ generó fragmentos de 20 y 97 pares de bases, mientras que el genotipo homocigoto para el alelo A generó un fragmento no digerido de 117 pares de bases. El polimorfismo -238G/A se determinó mediante la incubación con 2U de la enzima NLAIV (New England Biolabs). En este polimorfismo, el genotipo homocigoto para el alelo salvaje $G$ generó fragmentos de 47, 50 y 27 pares de bases, mientras que el genotipo homocigoto para el alelo A generó fragmentos de 47 y 70 pares de bases.

Para la determinación de los genotipos de los polimorfismos -376G/A y -163G/A, se amplificó un fragmento de 321 pares de bases usando los siguientes partidores: 5'-(clamp 25)- TTTTCCTGCATCCTGTCTG -3' (directo) and 5'- GCTCATCTGGAGGAAGCGGTAG -3' (reverso). Las condiciones de la reacción de PCR fueron iguales a las descritas en el párrafo anterior excepto en la temperatura de hibridación que fue en este caso de $59^{\circ} \mathrm{C}$. Los genotipos de los polimorfismos -376G/A y -163G/A fueron obtenidos mediante la incubación del producto de PCR con 2U de la enzima Tsp509I a 65으 (SNP -376G/A) o BstUI a 60ㄷ (-163G/A) (New England Biolabs), y posterior electroforesis en geles de agarosa de 3\% teñidos con bromuro de etidio. El genotipo homocigoto para el alelo $\mathrm{G}$ del polimorfismo -376G/A generó un fragmento no digerido de 321 pares de bases mientras que el genotipo homocigoto para el alelo A generó fragmentos de 46 y 275 pares de bases. Un total de ocho personas del estudio 1 no pudieron ser estudiadas para determinar el genotipo -376G/A por fallo repetido en la amplificación por PCR. Por otro lado, el genotipo homocigoto para el alelo $\mathrm{G}$ del polimorfismo -163G/A generó fragmentos de 261 y 60 pares de bases, mientras que el genotipo homocigoto para el alelo A generaría un fragmento no digerido de 321 pares de bases.

Estadística. Se calcularon las frecuencias alélicas y genotípicas por conteo directo y se evaluó la concordancia de las frecuencias genotípicas con el equilibrio genético de Hardy-Weinberg mediante un 
test exacto ${ }^{14}$. Las frecuencias haplotípicas y los tests de desequilibrio de ligamiento fueron calculados mediante el algoritmo de Esperanza-Maximización (EM) implementado en el paquete estadístico STATA 8.215. El índice $\mathrm{D}^{\prime}$ se calculó como medida de desequilibrio de ligamiento entre pares de SNP's ${ }^{16}$ usando los programas disponibles en la página web http://www-gene.cimr.cam.ac.uk/clayton/software.

Las variables continuas se expresaron como promedio \pm desviación estándar. Las diferencias entre los grupos de estudio fueron evaluadas con la prueba $\mathrm{t}$ de Student y técnicas de regresión lineal. Al evaluar la asociación entre variables categóricas, se utilizó el test exacto de Fisher.

\section{Resultados}

La Tabla 1 muestra las frecuencias alélicas y genotípicas de los cuatro polimorfismos estudiados, combinando la información de los estudios 1 y 2. Los polimorfismos G-308A, -238G/A y -376G/A presentaron frecuencias genotípicas concordantes con la ley de Hardy-Weinberg. La frecuencia del alelo A del SNP -163G/A es igual a cero por lo que este polimorfismo no fue incluido en los análisis posteriores.

Estudio 1. Se detectó un fuerte desequilibrio de ligamiento entre -238G/A y -376G/A ( $D^{\prime}=0,94 ; p$ $\varangle 0,001)$. Esto significa que cuando una persona era portadora del alelo A en la posición -376G/A, siempre era portadora del alelo A en -238G/A, existiendo cuatro personas heterocigotas únicamente en -238G/A sin serlo en -376G/A. No se detectó desequilibrio de ligamiento significativo entre los otros pares de polimorfismos ( $\mathrm{D}^{\prime}=0,33$ para $-238 \mathrm{G}$ / A y $-308 \mathrm{G} / \mathrm{A} ; \mathrm{D}^{\prime}=0,04$ para $-376 \mathrm{G} / \mathrm{A}$ y $\left.-308 \mathrm{G} / \mathrm{A}\right)$.
La Tabla 2 muestra la frecuencia de portadores del alelo A en las posiciones -308, -238 y -376 en relación con el estado de diabetes tipo 2. En esta Tabla, las frecuencias genotípicas mantuvieron la concordancia con la ley de Hardy-Weinberg en los diferentes grupos de estudio. No se encontraron diferencias significativas para la asociación entre polimorfismos de TNF-alfa y diabetes tipo 2, manteniéndose la falta de significación tras el ajuste por edad e IMC. Dado el alto desequilibrio de ligamiento encontrado entre 376G/A y 238G/A, la asociación -376A/G con diabetes sería equivalente a evaluar la asociación entre portadores del haplotipo de -376A y -238A con la diabetes.

No se encontraron diferencias relevantes al comparar portadores y no portadores del alelo $\mathrm{A}$ para los polimorfismos -308G/A y -238G/A, en relación con las variables de resistencia a la insulina en sujetos sin diagnóstico previo de diabetes (Tabla 3). Tampoco se encontraron diferencias significativas según los genotipos de -376G/A o los genotipos compuestos de -376G/A y -238G/A (datos no mostrados).

Estudio 2. La Tabla 4 muestra variables relacionadas con la adiposidad según los genotipos del polimorfismo -308G/A en las mujeres del estudio 2. De forma similar a la Tabla 3, los resultados de esta Tabla se restringen a las personas en las que no existía diagnóstico previo de diabetes. No se encontraron diferencias significativas en relación con el IMC, IMG o el porcentaje de grasa corporal (estimado según fórmula antropométrica validada). Se encontró una asociación casi significativa en el índice de cintura-cadera según los genotipos de $-308 G / A(p=0,06)$.

\section{Tabla 1. Frecuencia de cuatro polimorfismos de la región promotora de T N F-alfa en mujeres chilenas de 60-90 años de edad}

\begin{tabular}{|c|c|c|c|c|c|c|}
\hline & GG & $\begin{array}{l}\text { ecuen } \\
\text { genoti } \\
\text { GA }\end{array}$ & AA & $\begin{array}{l}\text { Tamaño } \\
\text { muestral }\end{array}$ & $\begin{array}{l}\text { Frecuencia } \\
\text { Alélica } \\
\text { (Alelo A) }\end{array}$ & $\begin{array}{c}\text { Valor p para la ley } \\
\text { de Hardy-Weinberg } \\
\text { (test exacto) }\end{array}$ \\
\hline SNP - $308 \mathrm{G} / \mathrm{A}$ & 0,81 & 0,19 & 0 & 263 & 0,09 & 0,14 \\
\hline $\mathrm{SNP}-238 \mathrm{G} / \mathrm{A}$ & 0,90 & 0,10 & 0 & 100 & 0,05 & 1,0 \\
\hline $\mathrm{SNP}-376 \mathrm{G} / \mathrm{A}$ & 0,93 & 0,07 & 0 & 92 & 0,03 & 1,0 \\
\hline SNP - $163 \mathrm{G} / \mathrm{A}$ & 1 & 0 & 0 & 100 & 0 & \\
\hline
\end{tabular}


Tabla 2.- D istribución de genotipos de la región promotora de T N F-alfa en mujeres chilenas (60-69 años) según su estado de diabetes tipo 2

\begin{tabular}{|c|c|c|c|c|}
\hline \multirow[b]{2}{*}{ Alelos y haplotipos } & \multicolumn{3}{|c|}{ Estado de diabetes tipo 2} & \multirow[b]{2}{*}{$\begin{array}{l}\text { Valor } \mathrm{p} \\
\text { (exacto) }\end{array}$} \\
\hline & Normal & $\begin{array}{l}\text { Intolerancia } \\
\text { a la glucosa }\end{array}$ & Diabetes & \\
\hline \multicolumn{5}{|c|}{ Posición -308G/A (Alelo -308A) } \\
\hline Portadores alelo A & $8(15 \%)$ & $1(6 \%)$ & $3(10 \%)$ & 0,71 \\
\hline No portadores & $45(85 \%)$ & $16(94 \%)$ & $27(90 \%)$ & \\
\hline Total & 53 & 17 & 30 & \\
\hline \multicolumn{5}{|c|}{ Posición -238G/A (Alelo -238A) } \\
\hline Portadores & $7(13 \%)$ & $1(6 \%)$ & $2(7 \%)$ & 0,66 \\
\hline No portadores & $46(87 \%)$ & $16(94 \%)$ & $28(93 \%)$ & \\
\hline Total & 53 & 17 & 30 & \\
\hline \multicolumn{5}{|c|}{ Posición -376G/A (Alelo -376A) ${ }^{\#}$} \\
\hline Portadores & $4(8 \%)$ & $1(6 \%)$ & $2(7 \%)$ & 0,73 \\
\hline No portadores & $44(92 \%)$ & $15(94 \%)$ & $26(93 \%)$ & \\
\hline Total & 48 & 16 & 28 & \\
\hline
\end{tabular}

\#El 100\% de las personas portadoras del alelo A en la posición -376 eran también portadoras del alelo A en la posición -238. Por tanto, y debido al alto desequilibrio de ligamiento entre ambos polimorfismos, los portadores del alelo A en -376 son, con casi total seguridad, portadores del haplotipo compuesto por los alelos -376A y 238A (es decir, el alelo A en ambas posiciones se encontranía en posición cis en un mismo haplotipo).

Tabla 3. M edia (desviación estándar) de variables relacionadas con la resistencia a la insulina y la obesidad en mujeres chilenas (60-69 años) sin diagnóstico previo de diabetes

\begin{tabular}{|c|c|c|c|c|c|c|}
\hline & \multicolumn{3}{|c|}{ Alelo -308A (SNP -308G/A) } & \multicolumn{3}{|c|}{ Alelo -238A (SNP -238G/A) } \\
\hline & $\begin{array}{c}\text { Portadores } \\
(\mathrm{n}=10)\end{array}$ & $\begin{array}{c}\text { No-portadores } \\
(\mathrm{n}=67)\end{array}$ & $\begin{array}{c}\mathrm{P}- \\
\text { value }\end{array}$ & $\begin{array}{l}\text { Portadores } \\
\quad(\mathrm{n}=8)\end{array}$ & $\begin{array}{c}\text { No-portadores } \\
(\mathrm{n}=69)\end{array}$ & Valor $\mathrm{p}$ \\
\hline $\begin{array}{l}\text { IMC } \\
\left(\mathrm{kg} / \mathrm{m}^{2}\right)\end{array}$ & $28,3(1,2)$ & $29,2(0,6)$ & 0,56 & $30,0(1,2)$ & $29,0(0,5)$ & 0,56 \\
\hline Cociente cintura-cadera & $0,85(0,01)$ & $0,88(0,02)$ & 0,10 & $0,82(0,04)$ & $0,86(0,01)$ & 0,14 \\
\hline Glucosa (mg/dl) & $93,0(4,2)$ & $98,9(2,4)$ & 0,37 & $94,5(2,9)$ & $98,5(2,4)$ & 0,58 \\
\hline Insulina (mU/ml) & $9,5(2,3)$ & $9,4(0,9)$ & 0,96 & $8,1(1,0)$ & $9,5(0,9)$ & 0,58 \\
\hline Índice HOMA-IR & $2,1(0,5)$ & $2,4(0,2)$ & 0,72 & $1,9(0,3)$ & $2,4(0,2)$ & 0,51 \\
\hline Colesterol (mg/dl) & $225,7(16,5)$ & $221,5(5,1)$ & 0,78 & $230,4(18,2)$ & $221,1(5,1)$ & 0,57 \\
\hline HDL-colesterol (mg/dl) & $44,6(4,3)$ & $42,3(1,5)$ & 0,59 & $44,0(4,4)$ & $42,5(1,5)$ & 0,74 \\
\hline Triglicéridos (mg/dL) & $144,2(20,8)$ & $156,2(8,5)$ & 0,61 & $190,9(33,2)$ & $150,5(7,9)$ & 0,12 \\
\hline
\end{tabular}

\#Los valores $p$ fueron calculados sobre la base de test $t$ de Student comparando portadores versus no-portadores en mujeres del estudio 1, sin diagnóstico previo de diabetes. El ajuste por edad entregó valores p similares a los mostrados en la Tabla 3.

\section{DisCUSIÓN}

Se han descrito numerosos polimorfismos en la región promotora del gen TNF-alfa humano que podrían ejercer un efecto sobre la expresión de este gen e impactar, de forma sensible, sobre diversas patologías de origen multifactorial ${ }^{1}$. Dado que TNF-alfa es un gen relacionado con la obesidad y la diabetes, se ha publicado un gran número de estudios epidemiológicos que han 
Tabla 4. M edia (desviación estándar) de variables relacionadas con la adiposidad en mujeres chilenas (60-90 años) sin diagnóstico previo de diabetes

\begin{tabular}{|c|c|c|c|}
\hline & \multicolumn{2}{|c|}{ Alelo -308A (SNP -308G/A) } & \multirow[b]{2}{*}{$\begin{array}{c}\text { Valor } \\
\mathrm{p}^{\#}\end{array}$} \\
\hline & $\begin{array}{l}\text { Portadores } \\
(\mathrm{n}=43)\end{array}$ & $\begin{array}{c}\text { No-portadores } \\
(\mathrm{n}=181)\end{array}$ & \\
\hline Índice de masa corporal $\left(\mathrm{kg} / \mathrm{m}^{2}\right)$ & $28,1(0,6)$ & $28,7(0,3)$ & 0,42 \\
\hline Cociente cintura-cadera & $0,87(0,01)$ & $0,86(0,004)$ & 0,06 \\
\hline Masa grasa (porcentaje) & $30,2(0,9)$ & $31,7(0,4)$ & 0,11 \\
\hline Índice de masa grasa $\left(\mathrm{kg}\right.$ grasa $\left./ \mathrm{m}^{2}\right)$ & $8,6(0,4)$ & $9,2(0,2)$ & 0,18 \\
\hline
\end{tabular}

\#Los valores $\mathrm{p}$ fueron calculados sobre la base de modelos de regresión lineal ajustados por edad en mujeres del estudio 2 sin diagnóstico previo de diabetes. El análisis no ajustado por edad entregó valores p muy similares a los mostrados en la Tabla 4.

intentado asociar estos polimorfismos con patologías relacionadas con componentes del síndrome metabólico múltiple ${ }^{17-32}$. Al igual que lo que ocurre en gran parte de los estudios de asociación en epidemiología genética de enfermedades complejas $^{33}$, los estudios que relacionan los polimorfismos de TNF-alfa con obesidad/diabetes han mostrado una gran discordancia en sus conclusiones $^{17-32}$. En un llamativo estudio de asociación, realizado en adultos mayores de origen holandés ${ }^{17}$, se estimó que el riesgo de diabetes asociado al genotipo homocigoto para el alelo A del polimorfismo -308G/A era más de 4,5 veces superior al riesgo de desarrollar diabetes en personas con el genotipo GG, lo que situaría a este polimorfismo como un importante determinante del desarrollo de diabetes tipo 2 en la población de adultos mayores.

Aunque existe aún debate sobre el papel de los polimorfismos de la región promotora de TNFalfa involucrados en la regulación de este gen ${ }^{34}$, existen numerosos estudios que sugieren un papel relevante del polimorfismo -308G/A sobre la expresión de TNF-alfa ${ }^{7,8}$. Específicamente, el alelo A en la posición -308 se ha relacionado con una mayor actividad de transcripción del gen de TNFalfa. Cuenca et $\mathrm{al}^{35}$ estimaron una frecuencia alélica de polimorfismo -308G/A similar a la encontrada en este estudio, y señalaron las importantes diferencias en las frecuencias alélicas, dependiendo del país y del grupo étnico estudiado. Teniendo en cuenta los antecedentes mencionados en estos dos últimos párrafos, se optó por investigar si efectivamente el polimorfismo -308G/ A de TNF-alfa podría ejercer un efecto mayor sobre la adiposidad y la distribución de grasa corporal en mujeres libres de diabetes.

El presente estudio tiene limitaciones que se derivan de la baja frecuencia de los alelos de los polimorfismos y de su naturaleza transversal. Sin embargo, es importante destacar que el diagnóstico de diabetes tipo 2 se realizó mediante una prueba oral de tolerancia a la glucosa, en contraste con otros estudios, en los que el diagnóstico de diabetes se basó en criterios más simples ${ }^{17}$. De los pacientes con diabetes, muchos de ellos ya conocían de su condición antes de iniciarse el estudio y algunos estaban ya bajo tratamiento farmacológico, al tiempo que habían modificado sus hábitos de alimentación en el pasado. Por ello, no hemos incluidos en las Tablas 3 y 4 a las personas con diagnóstico previo de diabetes, con el objeto de permitir una comparación no sesgada del impacto de los polimorfismos de TNF-alfa sobre las variables metabólicas y antropométricas relacionadas a la resistencia a la insulina (Tabla 3) y la adiposidad (Tabla 4).

En conclusión, es improbable que los polimorfismos de la región promotora de TNF-alfa tengan un impacto importante sobre la obesidad y la diabetes en la población chilena de adultos mayores de sexo femenino. Este reducido impacto puede concluirse, basándose en la baja frecuencia de los alelos de susceptibilidad descritos en la literatura y a la inexistencia de un efecto relevante de asociación genotipo-diabetes o genotipo-obesidad. 


\section{REFERENCIAS}

1. Aguilón JC, Cruzat A, Cuenca J, Cuchacovich T. El polimorfismo genético del factor de necrosis tumoral alfa como factor de riesgo en patología. Rev Méd Chile 2002; 130: 1043-50.

2. Uysal KT, Wiesbrock AM, Marino MW, Hotamisligil GS. Protection from obesity-induced insulin resistance in mice lacking TNF-alpha function. Nature 2002; 389: 610-14.

3. Hotamisligil GS, Shargill NS, Spiegelman BM. Adipose expression of tumor necrosis factor-alpha: direct role in obesity-linked insulin resistance. Science 1993; 259: 87-91.

4. Hotamisigil GS. Inflammatory pathways and insulin action. Int J Obes 2003; 27: S53-S55.

5. Dandona $\mathrm{P}$, Weinstock $R$, Thusu $\mathrm{K}$, Abdel-Rahman E, Aljada A, Wadden T. Tumor necrosis factor-alpha in sera of obese patients: fall with weight loss. J Clin Endocrinol Metab 1998; 83: 2907-10.

6. Patiño-García A, SotwLo-Piñeiro E, Modesto C, SierRaseSÚMAGA L Screening of the tumor necrosis factor-alpha $(\mathrm{NF}-\alpha)$ gene promoter polymorphisms by PCRDGGE analysis. Mut Res Genom 1999; 406: 121-5.

7. Wilson AG, Di Symons JA, McDowell TL, McDevitt HO, DuFF GW. Effects of a polymorphism in the human tumor necrosis factor $\alpha$ promoter on transcriptional activation. Proc Natl Acad Sci USA 1997; 94: 3195-9.

8. Abraham LJ, Kroeger KM. Impact of the -308 TNF promoter polymorphism on the transcriptional regulation of the TNF gene: relevance to disease. J Leukoc Biol 1999; 66: 562-6.

9. Santos Jl, Albala C, Lera L, García C, Arroyo P, Pérez-Bravo $\mathrm{F}$ et al. Anthropometric measurements in the elderly population of Santiago, Chile. Nutrition 2004; 20: 452-7.

10. Albala C, Yánez M, Salazar G, Vio F. Body composition in the elderly: total body water and anthropometry. Nutr Res 1994; 14: 1797-809.

11. Albala C, Salazar G, Yáñez M, Bunout D, Aicardi V, AgUiRRE E ET AL. Validation of an anthropometric model for total body water determination in the elderly. Nutr Res 1997; 17: 1-7.

12. Schutz Y, Kyle UUG, Pichard C. Fat-free mass index and fat mass index percentiles in Caucasians aged 18-98 y. Int J Obesity Relat Metab Disord 2002; 26: 953-60.

13. Carrasco EP, Pérez FB, Angel BB, Albala CB, Santos JL, Larenas GY et al. Prevalencia de diabetes tipo 2 y obesidad en dos poblaciones aborígenes de Chile en ambiente urbano. Rev Méd Chile 2004; 132: 1189-97.

14. Cleves M. Hardy-Weinberg equilibrium test and allele frequency estimation. Stat Tech Bull 1999; 48: 34-7.

15. MANDER AP. Haplotype analysis in populationbased association studies. STATA J 2001; 1: 58-75.

16. Devin B, Risch N. A comparison of linkage disequilibrium measures for fine-scale mapping. Genomics 1995; 29: 311-22.

17. Hejmans BT, Westendorp RG, Droog S, Kluft C, Knook DL, Stagboom PE. Association of the tumour necrosis factor alpha -308G/A polymorphism with the risk of diabetes in an elderly population-based cohort. Genes Immun 2002; 3: 225-8.

18. Brand E, Schorr U, Kunz I, Kertmen E, Ringel J, Distler A et al. Necrosis factor-alpha-308 G/A polymorphism in obese Caucasians. Int J Obes Relat Metab Disord 2001; 25: 581-5.

19. Corbalán MS, Martí A, Forga L, Patiño A, MartínEZ-González MA, Martínez JA. Influence of two polymorphisms of tumoral necrosis factor-alpha gene on the obesity phenotype. Diabetes Nutr Metab 2004; 17: 17-22.

20. De Silva AM, Waider KR, Boyko EJ, Whitecross KF, Nicholson G, Kотоwicz M Eт AL. Genetic variation and obesity in Australian women: a prospective study. Obes Res 2001; 9: 733-40.

21. Fernández-Real JM, GutiérRez C, Ricart W, Casamitjana R, Fernández-Castaner M, Vendrell J et al. The TNF alpha gene NcoI polymorphism influences the relationship among insulin resistance, percent body fat, and increased serum leptin concentrations. Diabetes 1997; 46: 1468-72.

22. Hermann SM, Ricard S, Nicaud V, Maluet C, Arveiler D, Evans A et aL. Polymorphisms of the tumour necrosis factor-alpha gene, coronary heart disease and obesity. Eur J Clin Invest 1998; 28: 59-66.

23. Ishi T, Hirose $H$, Saito I, Nishikai $K$, Maruyama $H$, SARUTA T. Tumor necrosis factor alpha gene 308G/Apolymorphism, insulin resistance, and fasting plasma glucose in young, older, and diabetic Japanese men. Metabolism 2000; 49: 1616-8.

24. Pihlajamaki J, Y linen M, Karhapaa P, Vauhronen I, LAaKso $M$. The effect of the -308 A allele of the TNF-alpha gene on insulin action is dependent on obesity. Obes Res 2003; 11: 912-7. 
25. Rasmussen SK, Urhammer SA, Jensen JN, Hansen T, Borch-Johnsen K, Pedersen O. The - 238 and 308 G.A polymorphisms of the tumor necrosis factor alpha gene promoter are not associated with features of the insulin resistance syndrome or altered birth weight in Danish Caucasians. J Clin Endocrinol Metab 2000; 85: 1731-4.

26. Romeo S, Sentineli F, Capici F, Arca M, Berni A, VECCI E ET AL. The -308G/A variant of the tumor necrosis factor alpha (TNF-alpha) gene is not associated with obesity, insulin resistance and body fat distribution. BMC Med Genet 2001; 2: 10.

27. Um JJ, Park JH, Kim HM. Gene polymorphisms in tumor necrosis factor locus and waist-hip ratio in obese Koreans. Clin Chim Acta 2003; 338: 11722.

28. Walston J, Seibert M, Yen CJ, Cheskin LJ, Andersen RE. Tumor necrosis factor alpha -238 and -308 polymorphisms do not associate with traits related to obesity and insulin resistance. Diabetes 1999; 48: 2096-8.

29. Hamman A, Mantzoros C, Vidal-Puig A, Fuer JS. Genetic variation in the TNF- $\alpha$ promoter is not associated with type II diabetes mellitus (NIDDM). Biochem Biophys Res Commun 1995; 211: 833-9.
30. Morris AM, Heilbronn LK, Noakes M, Kind KL, Cufton PM. -308 Nco polymorphism of tumour necrosis factor alpha in overweight Caucasians. Diab Res Clin Pract 2003; 62: 197-201.

31. Norman RA, Bogardus C, Ravussin E. Linkage between obesity and a marker near the tumor necrosis factor-alpha locus in Pima Indians. J Clin Invest 1995; 96: 158-62.

32. Kubaszek A, Pihlajamaki J, Komarovski V, Lindi V, LindSTROM J, ERIKSSON J et aL. Promoter polymorphisms of the TNF-alpha (G-308A) and IL-6 (C174G) genes predict the conversion from impaired glucose tolerance to type 2 diabetes: the Finnish Diabetes Prevention Study. Diabetes 2003; 52: 1872-6.

33. REDDEN DT, AwSON DB. Nonreplication in genetic association studies of obesity and diabetes research. J Nutr 2003; 133: 3323-6.

34. Bayley JP, OtTenhoff TH, VerweiJ CL. Is there a future for TNF promoter polymorphisms? Genes Immun 2004; 5: 315-29.

35. Cuenca J, Pérez CA, Aguirre AJ, Schiattino I, AguiLón JC. Genetic polymorphism at position308 in the promoter region of the tumor necrosis factor (TNF): implications of its allelic distribution on susceptibility or resistance to diseases in the Chilean population. Biol Res 2001; 34: 237-41. 